\title{
Schur complements in Krein spaces
}

\author{
A. Maestripieri and F. Martínez Pería
}

To the memory of Professor Mischa Cotlar

\begin{abstract}
The aim of this work is to generalize the notions of Schur complements and shorted operators to Krein spaces. Given a (bounded) $J$-selfadjoint operator $A$ (with the unique factorization property) acting on a Krein space $\mathcal{H}$ and a suitable closed subspace $\mathcal{S}$ of $\mathcal{H}$, the Schur complement $A_{/[\mathcal{S}]}$ of $A$ to $\mathcal{S}$ is defined. The basic properties of $A_{/[\mathcal{S}]}$ are developed and different characterizations are given, most of them resembling those of the shorted of (bounded) positive operators on a Hilbert space.
\end{abstract}

\section{Introduction}

Let $\mathcal{H}$ be a Hilbert space, $L(\mathcal{H})$ be the algebra of bounded linear operators on $\mathcal{H}$ and $L(\mathcal{H})^{+}$be the cone of positive operators in $L(\mathcal{H})$. Given $A \in L(\mathcal{H})^{+}$and a closed subspace $\mathcal{S}$ of $\mathcal{H}$, the Schur complement (or shorted operator) $A_{/ \mathcal{S}}$ was defined by M. G. Krein [16] and W. N. Anderson and G. E. Trapp [2] as

$$
A_{/ \mathcal{S}}=\max _{\leq}\left\{X \in L(\mathcal{H})^{+}: X \leq A, R(X) \subseteq \mathcal{S}^{\perp}\right\}
$$

where the natural order $\leq$ in $L(\mathcal{H})^{+}$is considered.

The notion of Schur complement was generalized to selfadjoint operators in Hilbert spaces, see [4], [9] ,[10], [17]. More generally, given Hilbert spaces $\mathcal{H}$ and $\mathcal{K}$, J. Antezana et. al. [6] defined the shorted operator for an arbitrary $A \in L(\mathcal{H}, \mathcal{K})$ with respect to a pair of suitable closed subspaces $\mathcal{S}$ and $\mathcal{T}$ of $\mathcal{H}$ ad $\mathcal{K}$, respectively.

If $A$ is a positive operator, E. Pekarev [18] proved that

$$
A_{/ \mathcal{S}}=A^{1 / 2} P_{\mathcal{M}^{\perp}} A^{1 / 2}
$$

where $\mathcal{M}=\overline{A^{1 / 2}(\mathcal{S})}$ and $P_{\mathcal{M}^{\perp}}$ is the orthogonal projection onto $\mathcal{M}^{\perp}$. This paper is devoted to study the Schur complement of $J$-selfadjoint operators in Krein spaces, whose definition is inspired by Eq. (1.1).

Let $\mathcal{H}$ be a Krein space with fundamental symmetry $J$. Bognár-Kramli's theorem [8] states that, if $A \in L(\mathcal{H})$ is $J$-selfadjoint then there exist a Krein space $\mathcal{K}$ and a bounded injective operator $D \in L(\mathcal{K}, \mathcal{H})$ such that

$$
A=D D^{\#},
$$

where $D^{\#} \in L(\mathcal{K}, \mathcal{H})$ denotes the $J$-adjoint operator of $D$. However, this decomposition may not be unique (see [19]). A $J$-selfadjoint operator $A \in L(\mathcal{H})$ has the unique factorization property if, for any pair of decompositions $A=D_{i} D_{i}^{\#}, D_{i} \in L\left(\mathcal{K}_{i}, \mathcal{H}\right), N\left(D_{i}\right)=\{0\}(i=1,2)$, there exists an isomorphism $U \in L\left(\mathcal{K}_{1}, \mathcal{K}_{2}\right)$ such that $D_{1}=D_{2} U$.

Consider a $J$-selfadjoint operator $A \in L(\mathcal{H})$ with the unique factorization property and suppose that $\mathcal{M}=\overline{D^{\#}(\mathcal{S})}$ is a Krein subspace of $\mathcal{K}$, then the Schur complement of $A$ to $\mathcal{S}$ is defined as

$$
A_{/[\mathcal{S}]}=D P_{\mathcal{M}[\perp] / / \mathcal{M}} D^{\#}
$$

where $\mathcal{M}^{[\perp]}$ is the $J$-orthogonal subspace to $\mathcal{M}$ in the Krein space $\mathcal{K}$ and $P_{\mathcal{M}^{[\perp]} / / \mathcal{M}} \in L(\mathcal{K})$ is the $J$-selfadjoint projection onto $\mathcal{M}^{[\perp]}$. 
The main properties of shorted operators in Hilbert spaces, which where proved by M. G. Krein [16], W. N. Anderson and G. E. Trapp [2] and E. Pekarev [18], have a natural counterpart for Schur complements in Krein spaces.

The contents of the paper are the following: Section 2 introduces the basic notation and some known results in Krein spaces including topics such as Bognár-Kramli's theorem, the unique factorization property, and $J$-contractive projections. It also contains the definition and a summary of the properties of the shorting operation in Hilbert spaces.

In Section 3, the Schur complement of $A$ to $\mathcal{S}, A_{/[\mathcal{S}]}$, and the $\mathcal{S}$-compression of $A, A_{[\mathcal{S}]}$, are defined for a given $J$-selfadjoint operator $A \in L(\mathcal{H})$ with the unique factorization property; also, the range and the nullspace of $A_{/[\mathcal{S}]}$ and $A_{[\mathcal{S}]}$ are characterized.

Section 4 is devoted to study the Schur complement for definite subspaces. In particular, it is proved that, if $\mathcal{M}=\overline{D^{\#}(\mathcal{S})}$ is a $J$-nonnegative subspace of $\mathcal{H}$, then

$$
A_{/[\mathcal{S}]}=\max _{\leq J}\left\{X \in \mathcal{I}(A): X \leq_{J} A, R(X) \subseteq \mathcal{S}^{[\perp]}\right\},
$$

where $\mathcal{I}(A)=\left\{X=E E^{\#}: E \in L(\mathcal{K}, \mathcal{H}), R(E) \subseteq R(D)\right\}$. Also, it is shown that

$$
A_{/[\mathcal{S}]}=\inf _{\leq J}\left\{Q^{\#} A Q: Q \in \mathcal{Q}(\mathcal{H}), N(Q)=\mathcal{S}\right\}
$$

Finally, in Section 5 the Schur complement for $J$-positive operators is described in detail. In this case $A_{/[\mathcal{S}]}$ is defined for every closed subspace $\mathcal{S}$ of $\mathcal{H}$ and it always has both extremal characterizations. Furthermore, the shorting operation of a $J$-positive operator $A$ in a Krein space $\mathcal{H}$ is intimately related to the shorted of $J A$ in the Hilbert space $|\mathcal{H}|$. This relationship allows to translate the classical results into the Krein space's context.

\section{Preliminaries}

Along this work $\mathcal{H}$ denotes either a (complex, separable) Hilbert space with inner product $\langle$,$\rangle or a$ (complex) Krein space with indefinite metric [, ], depending on the context. If $\mathcal{S}$ is a subspace of a Hilbert space $\mathcal{H}, \mathcal{S}^{\perp}$ is the orthogonal complement of $\mathcal{S}$. Analogously, if $\mathcal{S}$ is a subspace of a Krein space $\mathcal{H}$, the $J$-orthogonal subspace to $\mathcal{S}$ is the closed subspace of $\mathcal{H}$ defined by $\mathcal{S}^{[\perp]}=\{x \in \mathcal{H}:[x, y]=$ 0 for every $y \in \mathcal{S}\}$. Sometimes we use the notation $\mathcal{S}^{[\perp]_{\mathcal{H}}}$ instead of $\mathcal{S}^{[\perp]}$ to emphasize the Krein space considered.

Given two Hilbert spaces $\mathcal{H}$ and $\mathcal{K}, L(\mathcal{H}, \mathcal{K})$ is the algebra of bounded linear operators from $\mathcal{H}$ into $\mathcal{K}$ and $L(\mathcal{H})=L(\mathcal{H}, \mathcal{H})$. If $T \in L(\mathcal{H})$ then $T^{*}$ denotes the adjoint operator of $T, R(T)$ stands for the range of $T$ and $N(T)$ for its nullspace.

Given a Hilbert space $\mathcal{H}$, let $L(\mathcal{H})^{+}$be the cone of (semidefinite) positive operators in $L(\mathcal{H})$ and denote by $\mathcal{Q}(\mathcal{H})$ the set of projections in $L(\mathcal{H})$, i.e., $\mathcal{Q}(\mathcal{H})=\left\{Q \in L(\mathcal{H}): Q^{2}=Q\right\}$. If $\mathcal{S}$ and $\mathcal{T}$ are two (closed) subspaces of $\mathcal{H}$, denote by $\mathcal{S} \dot{+} \mathcal{T}$ the direct sum of $\mathcal{S}$ and $\mathcal{T}$. If $\mathcal{H}=\mathcal{S} \dot{+}$, the oblique projection onto $\mathcal{S}$ along $\mathcal{T}, P_{\mathcal{S} / / \mathcal{T}}$, is the projection with $R\left(P_{\mathcal{S} / / \mathcal{T}}\right)=\mathcal{S}$ and $N\left(P_{\mathcal{S} / / \mathcal{T}}\right)=\mathcal{T}$. In particular, $P_{\mathcal{S}}=P_{\mathcal{S} / / \mathcal{S}^{\perp}}$ is the orthogonal projection onto $\mathcal{S}$.

\section{Krein spaces}

In what follows we give some basic results on Krein spaces. For a complete exposition of the subject and the proofs of the results below see the books by J. Bognár [7] and T. Ya. Azizov and I. S. Iokhvidov [15], the monographs by T. Ando [3] and by M. Dritschel and J. Rovnyak [12] and the paper by J. Rovnyak [19].

Given a Krein space $\mathcal{H}$ and a fundamental decomposition $\mathcal{H}=\mathcal{H}_{+} \oplus \mathcal{H}_{-}$, the direct sum of the Hilbert spaces $\left(\mathcal{H}_{+},[],\right)$and $\left(\mathcal{H}_{-},-[],\right)$is denoted by $|\mathcal{H}|$. If $\mathcal{H}$ and $\mathcal{K}$ are Krein spaces then $L(\mathcal{H}, \mathcal{K})$ (respectively $L(\mathcal{H})$ ) stands for $L(|\mathcal{H}|,|\mathcal{K}|$ ) (respectively $L(|\mathcal{H}|)$ ). Given $T \in L(\mathcal{H}, \mathcal{K})$, the $J$-adjoint operator of $T$ is denoted by $T^{\#}$. An operator $T \in L(\mathcal{H})$ is $J$-selfadjoint if $T=T^{\#}$.

The following theorem is due to J. Bognár and A. Krámli [8]. See also Theorem 1.1 in [12]. 
Theorem 2.1 (Bognár-Krámli). Let $\mathcal{H}$ be a Krein space with fundamental symmetry J. Any J-selfadjoint operator $T \in L(\mathcal{H})$ can be written in the form

$$
T=W W^{\#},
$$

where $W \in L(\mathcal{K}, \mathcal{H})$ for some Krein space $\mathcal{K}$ and $N(W)=\{0\}$.

While factorizations as in Theorem 2.1 always exist, they are not in general unique.

Definition. Let $\mathcal{H}$ be a Krein space with fundamental symmetry $J$. A $J$-selfadjoint operator $T \in L(\mathcal{H})$ has the unique factorization property (UFP) if for any two factorizations

$$
T=W_{i} W_{i}^{\#}, \quad W_{i} \in L\left(\mathcal{K}_{i}, \mathcal{H}\right), \quad N\left(W_{i}\right)=\{0\}, \quad i=1,2,
$$

there is an isomorphism $U \in L\left(\mathcal{K}_{1}, \mathcal{K}_{2}\right)$ such that $W_{1}=W_{2} U$.

Remark 2.2. Let $T \in L(\mathcal{H})$ be a $J$-selfadjoint operator satisfying the UFP and suppose that $T=W W^{\#}$ where $W \in L(\mathcal{K}, \mathcal{H}), N(W)=\{0\}$ and $\mathcal{K}$ is a Krein space. Then,

1. if $T=D D^{\#}$ is another factorization of $T$ as in Theorem 2.1 then $R(D)=R(W)$;

2. if $R(T)$ is closed then $R\left(D^{\#}\right)=\mathcal{K}$.

An operator $T \in L(\mathcal{H})$ is $J$-positive if $[T x, x] \geq 0$ for every $x \in \mathcal{H}$. We denote it by $T \geq_{J} 0$. If $T_{1}$ and $T_{2}$ are $J$-selfadjoint operators, we say that $T_{1} \geq_{J} T_{2}$ if $T_{1}-T_{2} \geq_{J} 0$. It is easy to show that $\geq_{J}$ is a partial order in the real vector space of $J$-selfadjoint operators.

The following theorem provides some examples of classes of operators with the UFP.

Theorem 2.3. Let $\mathcal{H}$ be a Krein space with fundamental symmetry $J$, and let $T \in L(\mathcal{H})$ be a J-selfadjoint operator. Each of the following conditions is sufficient for $T$ to have the unique factorization property:

1. $T \geq_{J} 0$;

2. $T^{2} \leq_{J} T$.

Given a Krein space $\mathcal{H}$, an operator $T \in L(\mathcal{H})$ is $J$-contractive if $[T x, T x] \leq[x, x]$ for every $x \in \mathcal{H}$. Therefore, $T$ is $J$-contractive if and only if $T^{\#} T \leq_{J} I$. Analogously, an operator $T \in L(\mathcal{H})$ is $J$-expansive if $[T x, T x] \geq[x, x]$ for every $x \in \mathcal{H}$ (i.e. $T^{\#} T \geq_{J} I$ ).

We say that $\mathcal{S}$ is a Krein subspace of $\mathcal{H}$ if it is a Krein space with the indefinite metric of $\mathcal{H}$. It is well known that $\mathcal{S}$ is a Krein subspace of $\mathcal{H}$ if and only if $\mathcal{S}=R(Q)$ for some $J$-selfadjoint $Q \in \mathcal{Q}(\mathcal{H})$. Also, a subspace $\mathcal{S}$ of $\mathcal{H}$ is $J$-nonnegative (respectively $J$-nonpositive) if $[x, x] \geq 0$ (respectively $[x, x] \leq 0$ ) for every $x \in \mathcal{S}$.

S. Hassi and K. Nordström proved the following result, which characterizes those projections which are $J$-contractive (see $[14, \S 3$, Proposition 5]). A similar result holds for $J$-expansive projections.

Proposition 2.4. If $Q \in \mathcal{Q}(\mathcal{H})$ then the following conditions are equivalent:

1. Q is J-contractive;

2. $Q$ is J-selfadjoint and $N(Q)$ is J-nonnegative;

3. $I-Q$ is J-positive.

Hassi and Nordström $[14, \S 4$, Theorem 2] also proved that every $J$-selfadjoint projection $Q$ can be factored as follows.

Theorem 2.5. Let $Q$ be a J-selfadjoint projection in a Krein space $\mathcal{H}$. Then, $Q$ can be represented as $Q=Q_{+} Q_{-}$where $Q_{+}$and $Q_{-}$are two commuting projections such that $Q_{+}$is $J$-contractive and $Q_{-}$is $J$-expansive. 


\section{Shorted operators in Hilbert spaces}

Definition (Krein [16], Anderson-Trapp [1] [2]). Let $\mathcal{H}$ be a Hilbert space. Given $A \in L(\mathcal{H})^{+}$and a closed subspace $\mathcal{S}$ of $\mathcal{H}$, the shorted operator of $A$ to $\mathcal{S}$ is defined by

$$
A_{/ \mathcal{S}}=\max _{\leq}\left\{X \in L(\mathcal{H})^{+}: X \leq A, R(X) \subseteq \mathcal{S}^{\perp}\right\}
$$

where $\leq$ is the natural order given by the cone $L(\mathcal{H})^{+}$.

The following theorem collects many well known results about shorted operators. See [2], [18], [9], [10] for the proof of these facts.

Theorem 2.6. Let $\mathcal{S}$ be a closed subspace of a Hilbert space $\mathcal{H}$ and let $A \in L(\mathcal{H})^{+}$. Then:

1. If $\mathcal{M}=\overline{A^{1 / 2}(\mathcal{S})}$ then $A_{/ \mathcal{S}}=A^{1 / 2} P_{\mathcal{M}^{\perp}} A^{1 / 2}$.

2. $R(A) \cap \mathcal{S}^{\perp} \subseteq R\left(A_{/ \mathcal{S}}\right) \subseteq R\left(A^{1 / 2}\right) \cap \mathcal{S}^{\perp}$ and $N\left(A_{/ \mathcal{S}}\right)=A^{-1 / 2}(\mathcal{M})$.

3. $R\left(\left(A_{/ \mathcal{S}}\right)^{1 / 2}\right)=R\left(A^{1 / 2}\right) \cap \mathcal{S}^{\perp}$.

4. $A_{/ \mathcal{S}}=\inf \left\{Q^{*} A Q: Q \in \mathcal{Q}(\mathcal{H}), N(Q)=\mathcal{S}\right\}$.

5. If $\mathcal{T}$ is a closed subspace of $\mathcal{H}$ such that $\mathcal{S}+\mathcal{T}$ is closed then $A_{/ \mathcal{S}+\mathcal{T}}=\left(A_{/ \mathcal{S}}\right)_{/ \mathcal{T}}=\left(A_{/ \mathcal{T}}\right)_{/ \mathcal{S}}$.

If $\mathcal{H}$ is a Hilbert space and $\left(A_{n}\right)_{n \in \mathbb{N}}$ is a sequence in $L(\mathcal{H})$ we say that $\left(A_{n}\right)_{n \in \mathbb{N}}$ converges in the SOT topology to $A \in L(\mathcal{H})$ (and denote it by $A_{n} \underset{n \rightarrow \infty}{\stackrel{\text { sot }}{\longrightarrow}} A$ ) if $\left\|A_{n} x-A x\right\| \underset{n \rightarrow \infty}{\longrightarrow} 0$ for every $x \in \mathcal{H}$. Moreover, if $\left(A_{n}\right)_{n \in \mathbb{N}}$ and $A$ are selfadjoint operators, we say that $A_{n} \stackrel{\text { sot }}{\searrow} A$ if $A_{n} \underset{n \rightarrow \infty}{\stackrel{\text { sot }}{\longrightarrow}} A$ and $A_{n} \geq A_{n+1}(\geq A)$ for every $n \in \mathbb{N}$.

The following are some results about the continuity of the shorting operation, see [2], [5].

Proposition 2.7. Let $A_{n}(n \in \mathbb{N})$ and $A$ be operators in $L(\mathcal{H})^{+}$such that $A_{n} \searrow^{\text {SOT }} A$ as $n \rightarrow \infty$. Then, $\left(A_{n}\right)_{/ \mathcal{S}} \searrow^{S O T} A_{/ \mathcal{S}}$ as $n \rightarrow \infty$, for every closed subspace $\mathcal{S}$ of $\mathcal{H}$.

Proposition 2.8. Let $\mathcal{S}_{n}(n \in \mathbb{N})$ and $\mathcal{S}$ be closed subspaces such that $P_{\mathcal{S}_{n}}{ }^{\text {SOT }} P_{\mathcal{S}}$ as $n \rightarrow \infty$. Then, $A_{/ \mathcal{S}_{n}} \searrow^{\text {SOT }} A_{/ \mathcal{S}}$ as $n \rightarrow \infty$, for every $A \in L(\mathcal{H})^{+}$.

The following example shows that $P_{\mathcal{S}_{n}} \searrow^{\text {sot }} P_{\mathcal{S}}$ is not a sufficient condition to imply the convergence of the sequence $\left(A_{/ \mathcal{S}_{n}}\right)_{n \in \mathbb{N}}$ to $A_{/ \mathcal{S}}$.

Example 2.9. Let $A \in L(\mathcal{H})^{+}$such that $N(A)=\{0\}$ and $R(A)$ is not closed. Consider a dense subspace $\mathcal{T}$ of $\mathcal{H}$ such that $\mathcal{T} \cap R\left(A^{1 / 2}\right)=\{0\}$ and let $\left\{e_{n}\right\}_{n \in \mathbb{N}}$ be an orthonormal basis of $\mathcal{H}$ contained in $\mathcal{T}$.

Let $\mathcal{S}_{n}=\overline{\operatorname{span}\left\{e_{k}: k \geq n\right\}}$ for $n \geq 1$. Then, $P_{\mathcal{S}_{n}} \searrow^{\text {SOT }} 0$. Furthermore, $A_{/ \mathcal{S}_{n}}=0$ because

$$
R\left(\left(A_{/ \mathcal{S}_{n}}\right)^{1 / 2}\right)=R\left(A^{1 / 2}\right) \cap \mathcal{S}_{n}^{\perp}=R\left(A^{1 / 2}\right) \cap \operatorname{span}\left\{e_{1}, \ldots, e_{n}\right\}=\{0\} .
$$

But $A_{/\{0\}}=A \neq 0$.

\section{Schur complements in Krein spaces}

Let $\mathcal{H}$ be a Krein space with fundamental symmetry $J$ and $A \in L(\mathcal{H})$ be a $J$-selfadjoint operator satisfying the UFP. Suppose that $A=D D^{\#}$, where $\mathcal{K}$ is a Krein space and $D \in L(\mathcal{K}, \mathcal{H})$ with $N(D)=\{0\}$. Given a closed subspace $\mathcal{S}$ of $\mathcal{H}$, consider $\mathcal{M}=\overline{D^{\#}(\mathcal{S})}$ and suppose that $\mathcal{M}$ is a Krein subspace of $\mathcal{K}$. 
Definition. Under the above hypothesis, the Schur complement of $A$ to $\mathcal{S}$ is defined by

$$
A_{/[\mathcal{S}]}=D P_{\mathcal{M}[\perp] / / \mathcal{M}} D^{\#}
$$

and the $\mathcal{S}$-compression of $A$ is $A_{[\mathcal{S}]}=D P_{\mathcal{M} / / \mathcal{M}[\perp]} D^{\#}$.

The operators $A_{[\mathcal{S}]}$ and $A_{/[\mathcal{S}]}$ are well defined: by the UFP of $A$, if $A=D_{i} D_{i}^{\#}$ where $D_{i} \in L\left(\mathcal{K}_{i}, \mathcal{H}\right)$ and $N\left(D_{i}\right)=\{0\}$ for $i=1,2$, there exists an isomorphism $U \in L\left(\mathcal{K}_{1}, \mathcal{K}_{2}\right)$ such that $D_{1}=D_{2} U$. Given the subspaces $\mathcal{M}_{i}=\overline{D_{i}^{\#}(\mathcal{S})}$, for $i=1,2$, observe that $\mathcal{M}_{1}$ is a Krein subspace of $\mathcal{K}_{1}$ if and only if $\mathcal{M}_{2}=U\left(\mathcal{M}_{1}\right)$ is a Krein subspace of $\mathcal{K}_{2}$, and in this case $U P_{\mathcal{M}_{1} / / \mathcal{M}_{1}^{[\perp]}} U^{\#}=P_{\mathcal{M}_{2} / / \mathcal{M}_{2}^{[\perp]}}$. Then,

$$
D_{1} P_{\mathcal{M}_{1} / / \mathcal{M}_{1}^{[\perp]}} D_{1}^{\#}=D_{2}\left(U P_{\mathcal{M}_{1} / / \mathcal{M}_{1}^{[\perp]}} U^{\#}\right) D_{2}^{\#}=D_{2} P_{\mathcal{M}_{2} / / \mathcal{M}_{2}^{[\perp]}} D_{2}^{\#} \text {. }
$$

Also, the following properties hold for the Schur complement and the $\mathcal{S}$-compression:

i. $A_{[\mathcal{S}]}, A_{/[\mathcal{S}]} \in L(\mathcal{H})$,

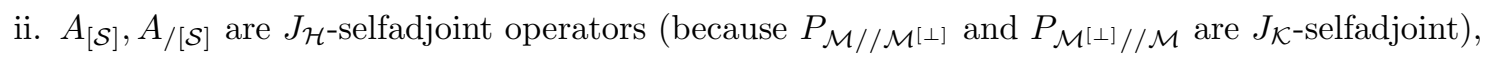

iii. $A_{[\mathcal{S}]}+A_{/[\mathcal{S}]}=A$.

Let us characterize the range and the nullspace of $A_{[\mathcal{S}]}$ and $A_{/[\mathcal{S}]}$. The lemma below is well known and its proof is straightforward.

Lemma 3.1. Let $\mathcal{H}$ and $\mathcal{K}$ be Krein spaces. If $T \in L(\mathcal{H}, \mathcal{K})$ then,

1. $N\left(T^{\#}\right)=R(T)^{[\perp]_{\mathcal{K}}}$.

2. $T^{\#}(\mathcal{S})^{[\perp]_{\mathcal{H}}}=T^{-1}\left(\mathcal{S}^{[\perp]_{\mathcal{K}}}\right)$ for every subspace $\mathcal{S}$ of $\mathcal{K}$.

Proposition 3.2. Let $A=D D^{\#} \in L(\mathcal{H})$ be a J-selfadjoint operator satisfying the UFP and $\mathcal{S}$ a closed subspace of $\mathcal{H}$ such that $\mathcal{M}=\overline{D^{\#}(\mathcal{S})}$ is a Krein subspace of $\mathcal{K}$. Then,

1. $A(\mathcal{S}) \subseteq R\left(A_{[\mathcal{S}]}\right) \subseteq \overline{A(\mathcal{S})}$;

2. $N\left(A_{[\mathcal{S}]}\right)=A(\mathcal{S})^{[\perp]}$;

3. $R(A) \cap \mathcal{S}^{[\perp]} \subseteq R(A /[\mathcal{S}]) \subseteq R(D) \cap \mathcal{S}^{[\perp]}$;

4. $N\left(A_{/[\mathcal{S}]}\right)=\left(D^{\#}\right)^{-1}(\mathcal{M})$.

Proof. 1. It is easy to see that

$$
A(\mathcal{S})=D\left(D^{\#}(\mathcal{S})\right)=A_{[\mathcal{S}]}(\mathcal{S}) \subseteq R\left(A_{[\mathcal{S}]}\right) \subseteq D(\mathcal{M})=D\left(\overline{D^{\#}(\mathcal{S})}\right) \subseteq \overline{D D^{\#}(\mathcal{S})}=\overline{A(\mathcal{S})}
$$

2. Since $N(D)=\{0\}$, it follows that

$$
N\left(A_{[\mathcal{S}]}\right)=N\left(P_{\mathcal{M} / / \mathcal{M}^{[\perp]}} D^{\#}\right)=\left(D^{\#}\right)^{-1}\left(\mathcal{M}^{[\perp]}\right)=A^{-1}\left(\mathcal{S}^{[\perp]}\right)=A(\mathcal{S})^{[\perp]} .
$$

3. First of all observe that, by Remark 2.2, $R(D)$ does not depend on the factorization. If $y \in$ $R(A) \cap \mathcal{S}^{[\perp]}$ then there exists $x \in \mathcal{H}$ such that $y=A x \in \mathcal{S}^{[\perp]}$. Note that $D^{\#} x \in \mathcal{M}^{[\perp]}$ and $A_{/[\mathcal{S}]} x=$ $D P_{\mathcal{M}[\perp] / / \mathcal{M}}\left(D^{\#} x\right)=D D^{\#} x=y$. Thus, $R(A) \cap \mathcal{S}^{[\perp]} \subseteq R\left(A_{/[\mathcal{S}]}\right)$. On the other hand, $R(A /[\mathcal{S}]) \subseteq$ $D\left(\mathcal{M}^{[\perp]}\right)=D\left(D^{-1}\left(\mathcal{S}^{[\perp]}\right)\right)=\mathcal{S}^{[\perp]} \cap R(D)$.

4. As in item 2., notice that $N\left(A_{/[\mathcal{S}]}\right)=N\left(P_{\mathcal{M}[\perp] / / \mathcal{M}} D^{\#}\right)=\left(D^{\#}\right)^{-1}(\mathcal{M})$.

In general, the inclusions in items 1 . and 3. of the above proposition are strict. See the examples in [2] and $[10]$.

Proposition 3.3. Let $A \in L(\mathcal{H})$ be a J-selfadjoint operator satisfying the UFP, $A=D D^{\#}, D \in L(\mathcal{K}, \mathcal{H})$ with $N(D)=\{0\}$, and $\mathcal{S}$ a closed subspace of $\mathcal{H}$ such that $\mathcal{M}=\overline{D^{\#}(\mathcal{S})}$ is a Krein subspace of $\mathcal{K}$. If $\mathcal{T}$ is a closed subspace of $\mathcal{H}$ such that $\mathcal{S} \subseteq \mathcal{T} \subseteq\left(D^{\#}\right)^{-1}(\mathcal{M})$ then $\overline{D^{\#}(\mathcal{T})}=\mathcal{M}$ and

$$
A_{/[\mathcal{T}]}=A_{/[\mathcal{S}]} \cdot
$$

Proof. Let $\mathcal{T}$ be a closed subspace of $\mathcal{H}$ such that $\mathcal{S} \subseteq \mathcal{T} \subseteq\left(D^{\#}\right)^{-1}(\mathcal{M})$, then applying $D^{\#}$ it follows that $D^{\#}(\mathcal{S}) \subseteq D^{\#}(\mathcal{T}) \subseteq D^{\#}\left(\left(D^{\#}\right)^{-1}(\mathcal{M})\right) \subseteq \mathcal{M}$. Therefore, $\overline{D^{\#}(\mathcal{T})}=\mathcal{M}$ and $A_{/[\mathcal{T}]}=A_{/[\mathcal{S}]}$. 


\section{Extremal properties for definite subspaces}

The main results in this section are stated for both $J$-nonnegative and $J$-nonpositive subspaces, but we only give the proofs for $J$-nonnegative ones. The proofs in the nonpositive case are similar.

Let $A \in L(\mathcal{H})$ be a $J$-selfadjoint operator satisfying the UFP. If $A=D D^{\#}$ where $\mathcal{K}$ is a Krein space and $D \in L(\mathcal{K}, \mathcal{H})$ with $N(D)=\{0\}$, consider the set

$$
\mathcal{I}(A)=\left\{X=E E^{\#}: E \in L(\mathcal{K}, \mathcal{H}), R(E) \subseteq R(D)\right\}
$$

By Remark 2.2, the subspace $R(D)$ only depends on $A$, so that, the same is true for the set $\mathcal{I}(A)$.

If $\mathcal{S}$ is a closed subspace of $\mathcal{H}$, consider the subsets

$$
\begin{aligned}
& \mathcal{M}^{-}\left(A, \mathcal{S}^{[\perp]}\right)=\left\{X \in \mathcal{I}(A): X \leq_{J} A, R(X) \subseteq \mathcal{S}^{[\perp]}\right\} \\
& \mathcal{M}^{+}\left(A, \mathcal{S}^{[\perp]}\right)=\left\{X \in \mathcal{I}(A): A \leq_{J} X, R(X) \subseteq \mathcal{S}^{[\perp]}\right\} .
\end{aligned}
$$

Observe that these sets can be empty.

First of all, consider the particular case $A=I$. Observe that $I \in L(\mathcal{H})$ has the UFP because it satisfies a sufficient condition: $I^{2}=I \leq_{J} I$ (see Theorem 2.3). Furthermore, the unique factorization (up to isomorphism) is $I=D D^{\#}$, where $D=I \in L(\mathcal{H})$ and therefore $\mathcal{M}^{-}\left(I, \mathcal{S}^{[\perp]}\right)=\left\{X \in L(\mathcal{H}): X \leq_{J}\right.$ $\left.I, R(X) \subseteq \mathcal{S}^{[\perp]}\right\}$ and $\mathcal{M}^{+}\left(I, \mathcal{S}^{[\perp]}\right)=\left\{X \in L(\mathcal{H}): I \leq_{J} X, R(X) \subseteq \mathcal{S}^{[\perp]}\right\}$.

Lemma 4.1. Let $\mathcal{S}$ be a Krein subspace of $\mathcal{H}$ and $Q=P_{\mathcal{S}}^{[\perp]} / / \mathcal{S}$. Then,

1. $Q=\max _{\leq J} \mathcal{M}^{-}\left(I, \mathcal{S}^{[\perp]}\right)$ if $\mathcal{S}$ is $J$-nonnegative.

2. $Q=\min _{\leq J} \mathcal{M}^{+}\left(I, \mathcal{S}^{[\perp]}\right)$ if $\mathcal{S}$ is J-nonpositive.

Proof. Suppose that $\mathcal{S}$ is a $J$-nonnegative Krein subspace of $\mathcal{H}$. Then, $Q$ is $J$-contractive (see Proposition 2.4) and $R(Q)=\mathcal{S}^{[\perp]}$. Therefore, $Q \in \mathcal{M}^{-}\left(I, \mathcal{S}^{[\perp]}\right)$.

Moreover, if $X \in \mathcal{M}^{-}\left(I, \mathcal{S}^{[\perp]}\right)$ then $X \leq_{J} Q: R(X) \subseteq \mathcal{S}^{[\perp]}$ implies that $Q X=X$, and $Q X Q=$ $(Q X) Q=X Q=Q X=X$ because $X$ and $Q$ are $J$-selfadjoint. Then, if $x \in \mathcal{H}$,

$$
[(Q-X) x, x]=[Q(I-X) Q x, x]=[(I-X) Q x, Q x] \geq 0,
$$

i.e. $X \leq_{J} Q$. Therefore, $Q=\max _{\leq J} \mathcal{M}^{-}\left(I, \mathcal{S}^{[\perp]}\right)$.

Corollary 4.2. Let $\mathcal{S}$ be a Krein subspace of $\mathcal{H}$. If $Q=P_{\mathcal{S}}^{[\perp] / / \mathcal{S}}$ then there exist two Krein subspaces $\mathcal{S}_{+}$and $\mathcal{S}_{-}$of $\mathcal{H}$ such that $\mathcal{S}=\mathcal{S}_{+}+\mathcal{S}_{-}$and

$$
Q=\max _{\leq J} \mathcal{M}^{-}\left(I, \mathcal{S}_{+}^{[\perp]}\right) \min _{\leq J} \mathcal{M}^{+}\left(I, \mathcal{S}_{-}^{[\perp]}\right)
$$

Proof. If $\mathcal{S}$ is a Krein subspace of $\mathcal{H}$ then, by Theorem 2.5, $Q=Q_{+} Q_{-}$, where $Q_{+}$and $Q_{-}$are commuting projections such that $Q_{+}$is $J$-contractive and $Q_{-}$is $J$-expansive. Also $\left(I-Q_{+}\right)\left(I-Q_{-}\right)=0$ (see the proof in [14]) so that $I-Q=\left(I-Q_{+}\right)+\left(I-Q_{-}\right)$and $\mathcal{S}=N(Q)=N\left(Q_{+}\right) \dot{+} N\left(Q_{-}\right)$.

By Lemma 4.1, $Q_{+}=\max _{\leq_{J}} \mathcal{M}^{-}\left(I, R\left(Q_{+}\right)\right)$and $Q_{-}=\min _{\leq_{J}} \mathcal{M}^{+}\left(I, R\left(Q_{-}\right)\right)$. Therefore, taking $\mathcal{S}_{ \pm}=N\left(Q_{ \pm}\right)$, the proof is complete.

The following theorem is an extremal characterization of the Schur complement similar to the one given by Anderson-Trapp [2, Theorem 1].

Theorem 4.3. Let $\mathcal{M}=\overline{D^{\#}(\mathcal{S})}$ be a Krein subspace of $\mathcal{K}$. Then:

$$
\begin{aligned}
& \text { 1. } A_{/[\mathcal{S}]}=\max _{\leq J} \mathcal{M}^{-}\left(A, \mathcal{S}^{[\perp]}\right) \text { if } \mathcal{M} \text { is J-nonnegative. } \\
& \text { 2. } A_{/[\mathcal{S}]}=\min _{\leq_{J}} \mathcal{M}^{+}\left(A, \mathcal{S}^{[\perp]}\right) \text { if } \mathcal{M} \text { is } J \text {-nonpositive. }
\end{aligned}
$$


Proof. Let $Q=P_{\mathcal{M}^{[\perp]} / / \mathcal{M}}$ and suppose that $\mathcal{M}$ is $J$-nonnegative (i.e. $Q$ is $J$-contractive). Notice that $A_{/[\mathcal{S}]}=(D Q)(D Q)^{\#}$ and $R(D Q) \subseteq R(D)$, then $A_{/[\mathcal{S}]} \in \mathcal{I}(A)$. Since $Q \leq_{J} I$ we have that $A_{/[\mathcal{S}]}=D Q D^{\#} \leq_{J} D D^{\#}=A$ and, by Proposition $3.2, R\left(A_{/[\mathcal{S}]}\right) \subseteq \mathcal{S}^{[\perp]}$. Therefore, $A_{/[\mathcal{S}]} \in \mathcal{M}^{-}\left(A, \mathcal{S}^{[\perp]}\right)$.

Moreover, $A_{/[\mathcal{S}]}=\max _{\leq J} \mathcal{M}^{-}\left(A, \mathcal{S}^{[\perp]}\right)$. Indeed, if $X=E E^{\#} \in \mathcal{M}^{-}\left(A, \mathcal{S}^{[\perp]}\right)$ then $R(E) \subseteq R(D)$ and, by Douglas' theorem [11, Theorem 1], the equation $D Y=E$ admits a bounded solution in $L(\mathcal{K})$. If $Z \in L(\mathcal{K})$ is a solution of the above equation, then $X=D Z Z^{\#} D^{\#}$. Since $X \leq_{J} A$, given $x \in \mathcal{H}$,

$$
\left[\left(I_{\mathcal{K}}-Z Z^{\#}\right) D^{\#} x, D^{\#} x\right]_{\mathcal{K}}=\left[D\left(I-Z Z^{\#}\right) D^{\#} x, x\right]_{\mathcal{H}}=[(A-X) x, x]_{\mathcal{H}} \geq 0,
$$

so $\left[\left(I_{\mathcal{K}}-Z Z^{\#}\right) y, y\right]_{\mathcal{K}} \geq 0$ for every $y \in \overline{R\left(D^{\#}\right)}=N(D)^{[\perp]_{\mathcal{K}}}=\mathcal{K}$. Hence, $Z Z^{\#} \leq{ }_{J} I_{\mathcal{K}}$. Since $R(X) \subseteq \mathcal{S}^{[\perp]}$ we have that $R\left(Z Z^{\#} D^{\#}\right) \subseteq D^{-1}\left(\mathcal{S}^{[\perp]}\right)=\mathcal{M}^{[\perp]}$. Moreover, $R\left(Z Z^{\#}\right)=Z Z^{\#}\left(\overline{R\left(D^{\#}\right)}\right) \subseteq \overline{R\left(Z Z^{\#} D^{\#}\right) \subseteq}$ $\mathcal{M}^{[\perp]}$. Therefore, $Z Z^{\#} \in \mathcal{M}^{-}\left(I, \mathcal{M}^{[\perp]}\right)$ and, by Lemma 4.1, $Z Z^{\#} \leq_{J} Q$ (notice that the Krein space considered here is $\mathcal{K}$ ). Then,

$$
X=D Z Z^{\#} D^{\#} \leq_{J} D Q D^{\#}=A_{/[\mathcal{S}]}
$$

i.e. $A_{/[\mathcal{S}]}=\max _{\leq J} \mathcal{M}^{-}\left(A, \mathcal{S}^{[\perp]}\right)$.

Corollary 4.4. Let $\mathcal{H}$ be a Krein space and $A \in L(\mathcal{H})$ a J-selfadjoint operator with the UFP. Consider a factorization $A=D D^{\#}$ where $\mathcal{K}$ is a Krein space and $D \in L(\mathcal{K}, \mathcal{H})$ with $N(D)=\{0\}$. If $A$ has closed range and $\mathcal{S}$ is a closed subspace of $\mathcal{H}$ such that $\mathcal{M}=\overline{D^{\#}(\mathcal{S})}$ is a Krein subspace of $\mathcal{K}$, then there exist two closed subspaces $\mathcal{S}_{+}$and $\mathcal{S}_{-}$of $\mathcal{H}$ such that $\mathcal{S}_{+}+\mathcal{S}_{-}=\left(D^{\#}\right)^{-1}(\mathcal{M})$ and

$$
A /[\mathcal{S}]=\max _{\leq J} \mathcal{M}^{-}\left(A, \mathcal{S}_{+}^{[\perp]}\right)+\min _{\leq J} \mathcal{M}^{+}\left(A, \mathcal{S}_{-}^{[\perp]}\right)-A
$$

Proof. Suppose that $\mathcal{M}$ is a Krein subspace of $\mathcal{K}$ and let $Q=P_{\mathcal{M}^{[\perp]} / / \mathcal{M}}$. By Theorem 2.5, there exist commuting projections $Q_{+}$and $Q_{-}$such that $Q=Q_{+} Q_{-}$, where $Q_{+}$is $J$-contractive, $Q_{-}$is $J$-expansive and $N(Q)=N\left(Q_{+}\right) \dot{+} N\left(Q_{-}\right)$(see the proof in [14]).

Let $\mathcal{S}_{ \pm}=\left(D^{\#}\right)^{-1}\left(N\left(Q_{ \pm}\right)\right)$and define $\mathcal{M}_{ \pm}=\overline{D^{\#}\left(\mathcal{S}_{ \pm}\right)}$. Since $R\left(D^{\#}\right)=\mathcal{K}$ (see Remark 2.2), it follows that $\mathcal{M}_{ \pm}=\overline{D^{\#}\left(\mathcal{S}_{ \pm}\right)}=\overline{N\left(Q_{ \pm}\right) \cap R\left(D^{\#}\right)}=N\left(Q_{ \pm}\right)$. Therefore, $A_{/\left[\mathcal{S}_{ \pm}\right]}=D Q_{ \pm} D^{\#}$ and

$$
A_{[\mathcal{S}]}=D(I-Q) D^{\#}=D\left(\left(I-Q_{+}\right)+\left(I-Q_{-}\right)\right) D^{\#}=A_{\left[\mathcal{S}_{+}\right]}+A_{\left[\mathcal{S}_{-}\right]} .
$$

As a consequence of Proposition 2.4, the subspaces $\mathcal{M}_{+}$and $\mathcal{M}_{-}$are $J$-nonnegative and $J$-nonpositive, respectively. Then, by Theorem 4.3 ,

$$
\begin{aligned}
A_{/[\mathcal{S}]} & =A-A_{[\mathcal{S}]}=A-\left(A_{\left[\mathcal{S}_{+}\right]}+A_{\left[\mathcal{S}_{-}\right]}\right)=A_{/\left[\mathcal{S}_{+}\right]}+A_{/\left[\mathcal{S}_{-}\right]}-A= \\
& =\max _{\leq_{J}} \mathcal{M}^{-}\left(A, \mathcal{S}_{+}^{[\perp]}\right)+\min _{\leq_{J}} \mathcal{M}^{+}\left(A, \mathcal{S}_{-}^{[\perp]}\right)-A .
\end{aligned}
$$

Theorem 4.5. Let $\mathcal{S}$ be a closed subspace of $\mathcal{H}$. Suppose that $A \in L(\mathcal{H})$ is J-selfadjoint and satisfies the UFP. If $A=D D^{\#}$ with $D \in L(\mathcal{K}, \mathcal{H}), N(D)=\{0\}$, suppose that $\mathcal{M}=\overline{D^{\#}(\mathcal{S})}$ is a Krein subspace of $\mathcal{K}$. Then:

1. $A_{/[\mathcal{S}]}=\inf _{\leq J}\left\{Q^{\#} A Q: Q \in \mathcal{Q}(\mathcal{H}), N(Q)=\mathcal{S}\right\}$ if $\mathcal{M}$ is $J$-nonnegative.

2. $A_{/[\mathcal{S}]}=\sup _{\leq J}\left\{Q^{\#} A Q: Q \in \mathcal{Q}(\mathcal{H}), N(Q)=\mathcal{S}\right\}$ if $\mathcal{M}$ is $J$-nonpositive.

Proof. Suppose that $\mathcal{M}$ is $J$-nonnegative and consider $P=P_{\mathcal{M}[\perp] / / \mathcal{M}}$. Then, for every $x \in \mathcal{K}$,

$$
[P x, P x]_{\mathcal{K}}=\min _{m \in \mathcal{M}}[x-m, x-m]_{\mathcal{K}}
$$

Indeed, given $x \in \mathcal{K}$ and $m \in \mathcal{M}$,

$[x-m, x-m]=[P x+(I-P) x-m, P x+(I-P) x-m]=[P x, P x]+[(I-P) x-m,(I-P) x-m] \geq[P x, P x]$. 
Furthermore, observe that $R\left(D^{\#}\right)$ is dense in $\mathcal{K}$ because $N(D)=\{0\}$. Then, if $y \in \mathcal{H}$,

$$
\begin{aligned}
{\left[A_{/[\mathcal{S}]} y, y\right]_{\mathcal{H}} } & =\left[P D^{\#} y, P D^{\#} y\right]_{\mathcal{K}}=\min _{m \in \mathcal{M}}\left[D^{\#} y-m, D^{\#} y-m\right]_{\mathcal{K}}=\inf _{s \in \mathcal{S}}\left[D^{\#}(y-s), D^{\#}(y-s)\right]_{\mathcal{K}}= \\
& =\inf _{s \in \mathcal{S}}[A(y-s), y-s]_{\mathcal{H}} .
\end{aligned}
$$

If $Q \in \mathcal{Q}(\mathcal{H})$ with $N(Q)=\mathcal{S}$, given $x \in \mathcal{H}$,

$$
\left[Q^{\#} A Q x, x\right]_{\mathcal{H}}=[A Q x, Q x]_{\mathcal{H}}=[A(x-(I-Q) x), x-(I-Q) x]_{\mathcal{H}} \geq\left[A_{/[\mathcal{S}]} x, x\right]_{\mathcal{H}}
$$

because $(I-Q) x \in \mathcal{S}$. Then, $A_{/[\mathcal{S}]} \leq_{J} Q^{\#} A Q$ for every $Q \in \mathcal{Q}(\mathcal{H})$ with $N(Q)=\mathcal{S}$ i.e. $A_{/[\mathcal{S}]}$ is a lower bound of the set $\left\{Q^{\#} A Q: Q \in \mathcal{Q}(\mathcal{H}), N(Q)=\mathcal{S}\right\}$.

Let $C$ be any lower bound of the set $\left\{Q^{\#} A Q: Q \in \mathcal{Q}(\mathcal{H}), N(Q)=\mathcal{S}\right\}$, we are going to show that $C \leq_{J} A_{/[\mathcal{S}]}$. Fixed $x \in \mathcal{H}$, if $x \notin \mathcal{S}$, observe that for every $s \in \mathcal{S}$ there exists $Q \in \mathcal{Q}(\mathcal{H})$ with $N(Q)=\mathcal{S}$ such that $(I-Q) x=s$. Therefore,

$$
[A(x-s), x-s]_{\mathcal{H}}=[A Q x, Q x]_{\mathcal{H}} \geq[C x, x]_{\mathcal{H}}
$$

for every $s \in \mathcal{S}$. Then, $\left[A_{/[\mathcal{S}]} x, x\right]_{\mathcal{H}} \geq[C x, x]_{\mathcal{H}}$. On the other hand, if $x \in \mathcal{S}$ then $Q^{\#} A Q x=0$ for every $Q \in \mathcal{Q}(\mathcal{H})$ with $N(Q)=\mathcal{S}$. Therefore,

$$
[C x, x]_{\mathcal{H}} \leq\left[Q^{\#} A Q x, x\right]_{\mathcal{H}}=0 .
$$

But $A_{/[\mathcal{S}]} x=D P_{\mathcal{M}[\perp] / / \mathcal{M}} D^{\#} x=0$ because $D^{\#} x \in \mathcal{M}$. Thus, $\left[A_{/[\mathcal{S}]} x, x\right]_{\mathcal{H}}=0 \geq[C x, x]_{\mathcal{H}}$. Since $x \in \mathcal{H}$ was arbitrary, $A_{/[\mathcal{S}]} \geq_{J} C$. So,

$$
A_{/[\mathcal{S}]}=\inf _{\leq J}\left\{Q^{\#} A Q: Q \in \mathcal{Q}(\mathcal{H}), N(Q)=\mathcal{S}\right\}
$$

\section{Schur complements of $J$-positive operators in Krein spaces}

By Theorem 2.3, $J$-positive operators have the unique factorization property. Furthermore, it is easy to see that, given a factorization as in Theorem 2.1, the vector space $\mathcal{K}$ acting as the domain of the factor can be chosen to be a Hilbert space (see Theorem 1.1 in [12]).

Let $\mathcal{H}$ be a Krein space and $A \in L(\mathcal{H})$ be $J$-positive. Along this section, we are going to use the following factorization of $A$ : if $|A|=J A \in L(|\mathcal{H}|)^{+}$, consider the Hilbert space $\mathcal{K}=J\left(N(A)^{\perp}\right)$ and $D=\left.J|A|^{1 / 2} J\right|_{\mathcal{K}} \in L(\mathcal{K}, \mathcal{H})$. Then, $N(D)=\{0\}, D^{\#}=J|A|^{1 / 2} \in L(\mathcal{H}, \mathcal{K})$ and $D D^{\#}=A$.

Observe that, if $\mathcal{K}$ is a Hilbert space and $\mathcal{S}$ is any closed subspace of $\mathcal{H}$, then the subspace $\mathcal{M}=\overline{D^{\#}(\mathcal{S})}$ is a closed subspace of $\mathcal{K}$ and therefore a "Krein subspace" of $\mathcal{K}$. Thus, the Schur complement $A_{/[\mathcal{S}]}$ is well defined for every closed subspace $\mathcal{S}$ of $\mathcal{H}$ and

$$
\begin{aligned}
A_{/[\mathcal{S}]} & =D P_{\mathcal{M}^{\perp}} D^{\#}=\left(J|A|^{1 / 2} J\right) P_{\mathcal{M}^{\perp}}\left(J|A|^{1 / 2}\right)=J|A|^{1 / 2}\left(J P_{\mathcal{M}^{\perp}} J\right)|A|^{1 / 2}= \\
& =J|A|^{1 / 2} P_{J\left(\mathcal{M}^{\perp}\right)}|A|^{1 / 2},
\end{aligned}
$$

where $P_{J\left(\mathcal{M}^{\perp}\right)} \in L(\mathcal{K})$ is the orthogonal projection onto $J\left(\mathcal{M}^{\perp}\right)$. Therefore, $A_{/[\mathcal{S}]}$ is $J$-positive. Furthermore, notice that the operator $E \in L\left(\mathcal{M}^{\perp}, \mathcal{H}\right)$ defined by $E x=D x=J|A|^{1 / 2} J x, x \in \mathcal{M}^{\perp}$ satisfies

$$
A_{/[\mathcal{S}]}=E E^{\#}, \quad \text { and } \quad N(E)=\{0\} .
$$

Therefore, it is the unique factorization (up to isomorphism) of $A_{/[\mathcal{S}]}$.

Remark 5.1. Observe that $J\left(\mathcal{M}^{\perp}\right)={\overline{J D^{\#}(\mathcal{S})}}^{\perp}=\left(|A|^{1 / 2}(\mathcal{S})\right)^{\perp}$. Thus, from Eq. (5.1) and item 1. of Theorem 2.6 follows that, if $A \in L(\mathcal{H})$ is $J$-positive then

$$
A_{/[\mathcal{S}]}=J\left(|A|_{/ \mathcal{S}}\right),
$$

where $|A|_{/ \mathcal{S}}$ is the shorted operator (in the Hilbert space sense) of $|A|$ to $\mathcal{S}$. 
Therefore, the shorting operation of a $J$-positive operator $A$ in a Krein space $\mathcal{H}$ is intimately related to the shorted of the positive operator $J A$ in the Hilbert space $|\mathcal{H}|$. The following propositions translate the classical results of Schur complements into Krein space's context. First of all, we state Douglas' theorem for $J$-positive operators in Krein spaces.

Theorem 5.2. Let $\mathcal{H}$ be a Krein space and consider J-positive operators $A, B \in L(\mathcal{H})$. If $A=D D^{\#}$, $D \in L\left(\mathcal{K}_{1}, \mathcal{H}\right), N(D)=\{0\}$ is any factorization of $A$ as in Theorem 2.1 (resp. $B=E E^{\#}, E \in L\left(\mathcal{K}_{2}, \mathcal{H}\right)$, $N(E)=\{0\})$ then the following conditions are equivalent:

1. equation $D X=E$ has a solution in $L\left(\mathcal{K}_{2}, \mathcal{K}_{1}\right)$;

2. $R(E) \subseteq R(D)$;

3. there exists $\lambda>0$ such that $B \leq_{J} \lambda A$.

In this case, there exists a unique $X \in L\left(\mathcal{K}_{2}, \mathcal{K}_{1}\right)$ such that $D X=E$. Moreover, $N(X)=N(E)$ and $\|X\|=\inf \left\{\lambda>0: B \leq_{J} \lambda A\right\}$.

Proof. Observe that if $A$ (resp. $B$ ) is $J$-positive then $\mathcal{K}_{1}$ (resp. $\mathcal{K}_{2}$ ) is a Hilbert space. Therefore, $D^{\#}=D^{*} J$ and $E^{\#}=E^{*} J$. So, equation $A \leq_{J} \lambda B$ is equivalent to $D D^{*} \leq \lambda E E^{*}$ and the results follows by Douglas' theorem [11].

Proposition 5.3. If $\mathcal{S}$ and $\mathcal{T}$ are closed subspaces of $\mathcal{H}$ and $A, B \in L(\mathcal{H})$ are $J$-positive, then

1. $A_{/[\mathcal{S}]}=\max _{\leq J} \mathcal{M}^{-}\left(A, \mathcal{S}^{[\perp]}\right)=\max _{\leq J}\left\{X \in L(\mathcal{H}): 0 \leq_{J} X \leq_{J} A, R(X) \subseteq \mathcal{S}^{[\perp]}\right\}$

2. $A_{/[\mathcal{S}]}=\inf _{\leq_{J}}\left\{Q^{\#} A Q: Q \in \mathcal{Q}(\mathcal{H}), N(Q)=\mathcal{S}\right\}$;

3. if $A \leq_{J} B$ then $A_{/[\mathcal{S}]} \leq_{J} B_{/[\mathcal{S}]}$

4. if $\mathcal{T} \subseteq \mathcal{S}$ then $A_{/[\mathcal{S}]} \leq_{J} A_{/[\mathcal{T}]}$.

Proof. 1. Given $A \in L(\mathcal{H}) J$-positive and $\mathcal{S}$ a closed subspace of $\mathcal{H}, A /[\mathcal{S}]=\max _{\leq J} \mathcal{M}^{-}\left(A, \mathcal{S}^{[\perp]}\right)$ by Theorem 4.3 (recall that $\mathcal{K}$ is a Hilbert space). Furthermore,

$$
\mathcal{M}^{-}\left(A, \mathcal{S}^{[\perp]}\right)=\left\{X \in L(\mathcal{H}): 0 \leq_{J} X \leq_{J} A, R(X) \subseteq \mathcal{S}^{[\perp]}\right\} .
$$

Let $\mathcal{A}=\left\{X \in L(\mathcal{H}): 0 \leq_{J} X \leq_{J} A, R(X) \subseteq \mathcal{S}^{[\perp]}\right\}$. If $X \in \mathcal{A}$ then $X \geq_{J} 0$ and it admits a factorization $X=E E^{\#}$, where $E \in L\left(\mathcal{K}_{1}, \mathcal{H}\right), N(E)=\{0\}$ and $\mathcal{K}_{1}$ is a Hilbert space, but we can substitute $\mathcal{K}_{1}$ be the Hilbert space $\mathcal{K}$ appearing in the decomposition of $A$. Since $X \leq_{J} A$ it follows that $R(E) \subseteq R(D)$ by Theorem 5.2. Thus $X \in \mathcal{I}(A)$, and the conditions $X \leq_{J} A$ and $R(X) \subseteq \mathcal{S}^{[\perp]}$ implies that $X \in \mathcal{M}^{-}\left(A, \mathcal{S}^{[\perp]}\right)$.

On the other hand, if $X \in \mathcal{M}^{-}\left(A, \mathcal{S}^{[\perp]}\right)$ then there exists $E \in L(\mathcal{K}, \mathcal{H})$ such that $X=E E^{\#}=E E^{*} J$ because $\mathcal{K}$ is a Hilbert space. Then, $X \geq_{J} 0$ and, by the remaining conditions on $X, X \in \mathcal{A}$. Therefore, $\mathcal{M}^{-}\left(A, \mathcal{S}^{[\perp]}\right) \subseteq \mathcal{A}$.

3. If $A \leq_{J} B$ then $|A|=J A \leq J B=|B|$. By Theorem 2.6, $|A|_{/ \mathcal{S}} \leq|B|_{/ \mathcal{S}}$ and therefore $A /[\mathcal{S}]=$ $J\left(|A|_{/ \mathcal{S}}\right) \leq_{J} J\left(|B|_{/ \mathcal{S}}\right)=B_{/[\mathcal{S}]}($ see Eq. $(5.2))$.

Items 2. and 4. follows analogously.

The following proposition generalizes item 3. of Theorem 2.6:

Proposition 5.4. Let $\mathcal{S}$ be a subspace of $\mathcal{H}$ and $A \in L(\mathcal{H})$ a J-positive operator. If $A=D D^{\#}$ (with $\mathcal{K}$ a Hilbert space, $D \in L(\mathcal{K}, \mathcal{H}), N(D)=\{0\}$ ) and $A_{/[\mathcal{S}]}=E E^{\#}$ (with $\mathcal{E}$ a Hilbert space, $E \in L(\mathcal{E}, \mathcal{H})$, $N(E)=\{0\}$ ) then

$$
R(E)=R(D) \cap \mathcal{S}^{[\perp]}
$$


Proof. If $A=D D^{\#}$ with $D \in L(\mathcal{K}, \mathcal{H}), N(D)=\{0\}$ then $A_{/[\mathcal{S}]}=F F^{\#}$ where $F \in L\left(\mathcal{M}^{\perp}, \mathcal{H}\right)$ is defined by $F x=D x$ for $x \in \mathcal{M}^{\perp}$. Thus,

$$
R(F)=R\left(D P_{\mathcal{M}^{\perp}}\right)=D\left(\mathcal{M}^{\perp}\right)=D\left(D^{-1}\left(\mathcal{S}^{[\perp]}\right)\right)=R(D) \cap \mathcal{S}^{[\perp]},
$$

and, by Remark 2.2, $R(E)=R(F)=R(D) \cap \mathcal{S}^{[\perp]}$.

Proposition 5.5. Let $\mathcal{H}$ be a Krein space and $A \in L(\mathcal{H})$ a $J$-positive operator. If $\mathcal{S}_{1}$ and $\mathcal{S}_{2}$ are closed subspaces of $\mathcal{H}$ such that $\mathcal{S}_{1}+\mathcal{S}_{2}$ is closed then

$$
A_{/\left[\mathcal{S}_{1}+\mathcal{S}_{2}\right]}=\left(A_{/\left[\mathcal{S}_{1}\right]}\right) /\left[\mathcal{S}_{2}\right]=\left(A_{/\left[\mathcal{S}_{2}\right]}\right) /\left[\mathcal{S}_{1}\right] .
$$

Proof. Suppose that $\mathcal{S}_{1}$ and $\mathcal{S}_{2}$ are closed subspaces of $\mathcal{H}$ such that $\mathcal{S}_{1}+\mathcal{S}_{2}$ is closed. Consider $|A|=$ $J A \in L(|\mathcal{H}|)^{+}$. Then, by item 4 . of Theorem 2.6, $|A|_{/ \mathcal{S}_{1}+\mathcal{S}_{2}}=\left(|A|_{/ \mathcal{S}_{1}}\right)_{/ \mathcal{S}_{2}}=\left(|A|_{/ \mathcal{S}_{2}}\right) / \mathcal{S}_{1}$. Therefore, by Eq. (5.2),

$$
A_{/\left[\mathcal{S}_{1}+\mathcal{S}_{2}\right]}=J\left(|A|_{/ \mathcal{S}_{1}+\mathcal{S}_{2}}\right)=J\left[\left(|A|_{/ \mathcal{S}_{1}}\right)_{/ \mathcal{S}_{2}}\right]=\left(J\left(|A|_{/ \mathcal{S}_{1}}\right)\right)_{/\left[\mathcal{S}_{2}\right]}=\left(A_{/\left[\mathcal{S}_{1}\right]}\right)_{/\left[\mathcal{S}_{2}\right]} .
$$

Analogously, $A_{/\left[\mathcal{S}_{1}+\mathcal{S}_{2}\right]}=\left(A_{/\left[\mathcal{S}_{2}\right]}\right)_{/\left[\mathcal{S}_{1}\right]}$.

In what follows, given a sequence $\left(T_{n}\right)_{n \in \mathbb{N}}$ of $J$-positive operators, the notation $T_{n} \stackrel{\text { J-SOT }}{\searrow} T$ stands for $T_{n} \underset{n \rightarrow \infty}{\stackrel{\text { soT }}{\longrightarrow}} T$ and $T_{n} \geq_{J} T_{n+1}\left(\geq_{J} T\right)$ for every $n \in \mathbb{N}$.

Observe that, $T_{n} \searrow^{\text {J-sot }} T$ if and only if $J T_{n} \searrow^{\text {SOT }} J T$ : Indeed, if $T_{n} \stackrel{\text { J-sot }}{\searrow} T$ then $T_{n} \underset{n \rightarrow \infty}{\stackrel{\text { sot }}{\longrightarrow}} T$ and $T_{n} \geq_{J} T_{n+1}\left(\geq_{J} T\right)$. Equivalently, $J T_{n} \underset{n \rightarrow \infty}{\stackrel{\text { sot }}{\longrightarrow}} J T$ (because $J$ is invertible) and $J T_{n} \geq J T_{n+1}(\geq J T)$, i. e. $J T_{n} \searrow^{\text {sОT }} J T$.

The next proposition follows easily using the remark above and Propositions 2.7 and 2.8 .

Proposition 5.6. Let $\mathcal{H}$ be a Krein space.

1. If $\left(A_{n}\right)_{n \in \mathbb{N}}$ is a sequence of $J$-positive operators in $L(\mathcal{H})$ such that $A_{n} \stackrel{\text { J-SOT }}{\searrow} A$, then

$$
A_{n /[\mathcal{S}]} \searrow^{\text {J-SOT }} A_{/[\mathcal{S}]} .
$$

2. If $\left(\mathcal{S}_{n}\right)_{n \in \mathbb{N}}$ and $\mathcal{S}$ are closed subspaces of $\mathcal{H}$ such that $\mathcal{S}_{n} \subseteq \mathcal{S}_{n+1}$ for every $n \in \mathbb{N}$ and $\mathcal{S}=\overline{\bigcup_{n \in \mathbb{N}} \mathcal{S}_{n}}$, then $A_{/\left[\mathcal{S}_{n}\right]} \searrow^{\text {J-SOT }} A_{/[\mathcal{S}]}$ for every $J$-positive operator $A \in L(\mathcal{H})$.

Remark 5.7. Example 2.9 can be modified to prove that item 2 of Proposition 5.6 is not true if $\mathcal{S}_{n} \supseteq \mathcal{S}_{n+1}$ for every $n \in \mathbb{N}$ and $\mathcal{S}=\bigcap_{n \in \mathbb{N}} \mathcal{S}_{n}$.

\section{Acknowledgment}

The authors would like to acknowledge Jorge A. Antezana for fruitful comments concerning shorted operators in Hilbert spaces and the results herein. They also express their gratitude to the referee who helped them to improve this paper.

\section{References}

[1] W. N. Anderson Jr., Shorted operators, SIAM J. Appl. Math. 20 (1971) 520-525.

[2] W. N. Anderson Jr. and G. E. Trapp, Shorted operators II, SIAM J. Appl. Math. 28 (1975) 60-71.

[3] T. Ando, Linear operators on Krein spaces, Hokkaido University, Sapporo, Japan, 1979. 
[4] T. Ando, Generalized Schur complements, Linear Algebra Appl. 27 (1979), 173-186.

[5] J. Antezana, G. Corach and D. Stojanoff, Spectral Shorted Operators, Integ. equ. oper. theory 55 (2006), 169-188.

[6] J. Antezana, G. Corach and D. Stojanoff, Bilateral shorted operators and parallel sums, Linear Algebra Appl. 414 (2006), no. 2-3, 570-588.

[7] J. Bognár, Indefinite inner product spaces, Springer-Verlag, 1974.

[8] J. Bognár and A. Krámli, Operators of the form $C^{*} C$ in indefinite inner product spaces, Acta Sci. Math. (Szeged) 29 (1968), 19-29.

[9] G. Corach, A. Maestripieri and D. Stojanoff, Oblique projections and Schur complements, Acta Sci. Math. (Szeged) 67 (2001), 337-256.

[10] G. Corach, A. Maestripieri and D. Stojanoff, Generalized Schur complements and oblique projections, Linear Algebra Appl. 341 (2002), 259-272.

[11] R. G. Douglas, On majorization, factorization and range inclusion of operators in Hilbert space, Proc. Amer. Math. Soc. 17 (1966), 413-416.

[12] M. A. Dritschel and J. Rovnyak, Operators on indefinite inner product spaces, Fields Institute Monographs no. 3, Amer. Math. Soc. Edited by Peter Lancaster 1996, 3, 141-232.

[13] M. A. Dritschel and J. Rovnyak, Extension theorems for contraction operators on Krein spaces, Oper. Theory Adv. Appl. 47 (1990), 221-305.

[14] S. Hassi and K. Nordström, On projections in a space with an indefinite metric, Linear Algebra Appl. 208-209 (1994), 401-417.

[15] I. S. Iokhvidov, T. Ya. Azizov, Linear Operators in spaces with an indefinite metric, John Wiley and sons, 1989.

[16] M. G. Krein, The theory of self-adjoint extensions of semibounded Hermitian operators and its applications, Mat. Sb. (N. S.) 20 (62) (1947), 431-495.

[17] P. Massey and D. Stojanoff, Generalized Schur Complements and P-Complementable Operators, Linear Algebra Appl. 393 (2004) 299-318.

[18] E. L. Pekarev, Shorts of operators and some extremal problems, Acta Sci. Math. (Szeged) 56 (1992) $147-163$.

[19] J. Rovnyak, Methods on Krein space operator theory, Interpolation theory, systems theory and related topics (Tel Aviv/Rehovot, 1999), Oper. Theory Adv. Appl., 134 (2002), 31-66. 